\title{
Differential game of optimal pursuit for an infinite system of differential equations
}

\begin{abstract}
We study an optimal pursuit differential game problem in the Hilbert space $12 \mathrm{r}+1$. The game is described by an infinite system of the first-order differential equations whose coefficients are negative. The control functions of players are subjected to integral constraints. If the state of the system coincides with the origin of the space $12 \mathrm{r}+1$, then game is considered completed. We obtain an equation to find the optimal pursuit time. Moreover, we construct the optimal strategies for players.
\end{abstract}

Keyword: Differential game; Infinite system; Pursuer; Evader; Hilbert space; Integral constraint; Optimal strategy 